\title{
La importancia de la Educación Ambiental en estudiantes de básica $y$ media en tres instituciones educativas públicas en El Espinal (Tolima) ${ }^{1}$
}

The importance of Environmental Education in
basic and high school students in three public
educational institutions in El Espinal (Tolima)

(D) Julián Enrique Barrero García ${ }^{2}$

Cómo citar este artículo:

Recepción: Mayo 11 de 2020 Aprobación: Junio 24 de 2020

Publicación: Junio 30 de 2020

Barrero G, Julián (2020). "La Importancia de la Educación Ambiental en Estudiantes de Básica y Media en Tres Instituciones Educativas Públicas en El Espinal (Tolima)".

Miradas, Vol. 15, Na 1.pp. 129 - 142

https://doi.org/10.22517/25393812.24473

Resumen

El presente artículo de reflexión realiza un análisis sobre la importancia que tiene la Educación Ambiental para los estudiantes de educación básica y media en tres instituciones educativas públicas de El Espinal (Tolima), el papel y rol que esta cumple para su vida personal y académica, comprendiendo la intervención que esta educación puede realizar en la construcción

1 Proyecto de Investigación: Estado Actual de la Educación Ambiental en Tres Instituciones Educativas Públicas de Básica y Media de El Espinal (Tolima). Trabajo de Grado para la Maestría en Educación Ambiental de la Universidad del Tolima.

2 Ingeniero Industrial. Especialista en Gestión Ambiental. Magister en Educación Ambiental. Docente Investigador. Escuela Militar de Suboficiales "Sargento Inocencio Chincá". Ejército Nacional de Colombia. Julian.barrero.profesor@emsub.edu.co ORCID 0000-0003-2143-9461 
y transformación social desde la enseñanza de valores para una buena relación con el ambiente, crear hábitos y comportamientos que permitan mejorar los conflictos y problemáticas ambientales presentes en la sociedad actual y el educar para proteger, cuidar y preservar los recursos naturales para las generaciones presentes y futuras. Este análisis se llevó a cabo a través de la aplicación de una encuesta a estudiantes de los grados noveno, decimo y undécimo.

Se encuentra que los temas ambientales son asignados al área de Ciencias Naturales, los docentes no tienen una formación ambiental y no se presenta una transversalidad de la Educación Ambiental en el plan de estudios y esta se limita a la celebración de fechas ambientales en el calendario académico, para los estudiantes lo ambiental y la Educación Ambiental es importante para su vida académica y personal presentando un compromiso e interés.

Palabras Clave: Educación

Ambiental, Cultura Ambiental,

Educación, Estudiante, Docente

\section{Abstract}

This reflection article analyzes the importance of Environmental Education for elementary and middle school students in three public educational institutions in El Espinal (Tolima), the role and role it plays in their personal and academic life, understanding the intervention that this education can carry out in the construction and social transformation from the teaching of values for a good relationship with the environment, creating habits and behaviors that allow improving the conflicts and environmental problems present in today's society and educating to protect, care for and preserve natural resources for present and future generations. This analysis was carried out through the application of a survey to students in the ninth, tenth and eleventh grades.

It is found that environmental issues are assigned to the area of Natural Sciences, teachers do not have environmental training and there is no crosscutting of Environmental Education in the study plan and this is limited to the celebration of environmental dates in the academic calendar, for students, environmental and Environmental Education is important for their academic and personal life, presenting a commitment and interest.

Keywords: Environmental Education, Environmental Culture, Education, Student, Teacher

\section{Introducción}

En la actualidad la humanidad enfrenta diferentes conflictos y problemáticas ambientales que giran en torno a ese desarrollo propuesto por la modernidad, que trae consigo la movilidad de los diferentes sectores de la economía, lo cual ha generado la pérdida de biodiversidad, la sobrepoblación, la contaminación, la deforestación, el calentamiento global y la desmesurada extracción de los recursos naturales para la generación 
de bienes y servicios, causando daños y alteraciones a los ecosistemas.

La educación ambiental
debe entenderse
como un proceso de
aprendizaje que debe
facilitar la comprensión
de las realidades del
medioambiente, del
proceso socio histórico
que ha conducido a su
actual deterioro; que
tiene como propósito que
cada individuo posea una
adecuada conciencia de
dependencia y pertenencia
con su entorno, que se
sienta responsable de su uso
y mantenimiento, y que sea
capaz de tomar decisiones
en este plano. (Alea, 2008,
pág. 3.).

La Educación Ambiental es uno de los pilares y labase que puede permitir el desarrollo de las comunidades, ya que a través de este proceso educativo se puede realizar un cambio de pensamiento y comportamiento para el cuidado y preservación del ambiente y la biodiversidad. En las instituciones educativas existen múltiples espacios de aprendizaje desde los cuales se puede desarrollar la Educación Ambiental, ya sea inmersa en el currículo para ser trabajada desde el aula en las diferentes asignaturas, en la propuesta y ejecución de Proyecto Ambiental Escolar (PRAE) o si no se presenta transversalidad se puede abordar desde el área de Ciencias Naturales. Desafortunadamente, se encuentra la realidad de que la Educación Ambiental es mencionada en algunas ocasiones en temas dentro de los planes de estudios en las instituciones educativas para dar cumplimiento de la normatividad, no para profundizar en la reflexión, el pensamiento crítico y la complejidad que se podría trabajar entre el docente y los estudiantes frente al análisis de las distintas problemáticas y conflictos ambientales presentes específicamente en el territorio en donde se encuentre la institución educativa, teniendo claro que todos los territorios presentan situaciones diferentes. En ese sentido:

La enseñanza r sobre
temas ambientales tiene
un carácter limitado
que se asocia con la
asignatura Ciencias
Naturales, desconociendo
la importancia sistémica,
que requiere de un proceso
pedagógico integral que
lleve al estudiante y demás
miembros de la comunidad
académica, al desarrollo de
actividades preventivas y
no reactivas. (Sepúlveda-
Gallego, 2007).

Frente a los retos actuales que se presentan en el planeta, la implementación de una Educación Ambiental desde lo integral y lo complejo en las instituciones educativas permitirá que se desarrollen en el estudiante competencias, habilidades, destrezas, hábitos y comportamientos que permitan a través del proceso pedagógico abordar la construcción de una nueva realidad con miras a la transformación social entorno a las diferentes realidades que vive el país y el mundo. 
La educación ambiental en Colombia se ha venido posicionando como una invitación a reinventar el papel de padres, maestros, alumnos, trabajadores, vecinos, gestores $\mathrm{y}$ tomadores de decisiones, entre otros, a perfilar una ética de la convivencia y de la responsabilidad; una ética ciudadana que reconozca la pluralidad (nuestro carácter multiétnico), $\mathrm{y}$, por consiguiente, facilite una comunicación fértil y fluida, que contribuya de manera importante en la apertura de caminos y aproximaciones a la sostenibilidad ambiental y a los cambios fundamentales que hoy requiere el país. (Carrasco, 2010).

El presente artículo de reflexión realiza un análisis sobre la importancia que tiene la Educación Ambiental para los estudiantes de educación básica y media en tres instituciones educativas públicas de El Espinal (Tolima); comprendiendo el interés y compromiso que tienen las generaciones actuales para con el ambiente, se realiza una encuesta a estudiantes de grado noveno, décimo y undécimo, reflexionando, analizando y estableciendo diferentes puntos de vista desde el pensamiento juvenil en una relación hombre naturaleza que a futuro pueda ser mejor que la que se tiene actualmente. Se reconocen otros actores que pueden intervenir en fortalecer estos procesos de aprendizaje desde lo ambiental para seguir generando conciencia y cultura ambiental en los jóvenes de hoy.

Todo proceso o programa
de educación ambiental
debe tener en cuenta las
características de las
personas en relación con
el ciclo vital, la cultura y,
en general, el contexto en
donde se van a desarrollar
para adecuar de manera
pertinente las acciones
o estrategias. (Vargas \&
Estupiñán, 2012).

Las instituciones educativas seleccionadas fueron tres, dos del sector urbano y una del sector rural, las cuales serán identificadas como Institución Educativa Sector Urbano 1 (IESU1), Institución Educativa Sector Urbano 2 (IESU2) e Institución Educativa Sector Rural 3 (IESR3); de acuerdo a su propuesta educativa institucional, establecen dentro del Proyecto Educativo Institucional (PEI) la formación del individuo con conciencia y cultura ambiental, basado en el respeto, responsabilidad, cuidado y preservación del ambiente y los seres vivos que hacen parte de él. Reconociendo las problemáticas y conflictos ambientales que se presentan en el territorio y la forma de poder intervenir en ellos desde la academia. Formando una sociedad en la que se aprovechen los recursos naturales presentes pero pensando de igual manera en las generaciones futuras. Se espera identificar las buenas prácticas educativas para logar una Educación Ambiental con fines de transformación social. 


\section{Desarrollo}

\section{Educación Ambiental y Escuela}

La Educación Ambiental a nivel general puede ser entendida, comprendida y vista como un camino y una guía hacia la intervención y solución de las problemáticas y conflictos ambientales que se presentan en los diferentes territorios en donde hacen presencia diferentes organizaciones sociales e instituciones educativas que son los lugares en donde las personas desarrollan las personas sus relaciones humanas. Pero no solamente estas relaciones, también al estar en contacto con el ambiente y con el entorno tienen relación con otros seres vivos que lo habitan. Por esto mismo, la Educación Ambiental brinda acciones y permite fortalecer los procesos pedagógicos en los estudiantes para que estos tengan una relación armoniosa con el ambiente y lo habiten de una manera no dañina. Desde esta educación, se promueve la generación de una cultura y conciencia ambiental para cuidar, preservar, transformar $y$ valorar los recursos naturales que el ambiente suministra para satisfacer las necesidades básicas de las generaciones presentes sin olvidar las futuras.

La EA es un proceso educativo, integral e interdisciplinario que considera al ambiente como un todo y que busca involucrar a la población en general en la identificación y la resolución de problemas a través de la adquisición de conocimientos, valores, actitudes y habilidades, la toma de decisiones y la participación organizada (Romero, 1997).

En Colombia la Educación Ambiental en las instituciones educativas esta legislada desde 1994 a través del Proyecto Ambiental Escolar (PRAE), el cual es un proyecto transversal que deben realizar todas las instituciones educativas dentro de su Proyecto Institucional (PEI), pero que desafortunadamente no tiene el éxito y el cumplimiento de los objetivos para los cuales en verdad debe ser propuesto, ya que en la mayoría de los casos se realiza solo para cumplir el requisito de la norma, a pesar de ser un proyecto importante que permite la integración e interacción de todos los integrantes de la comunidad educativa en la propuesta, diseño, elaboración e intervención de necesidades y situaciones ambientales que se puedan mejorar o en las que se pueda ver inmersa toda esta comunidad fortaleciendo la relación con el ambiente, a través de actividades, programas, jornadas y demás que giren en torno a ciertos temas ambientales y también sociales en los cuales se pueda desarrollar desde la Educación Ambiental un proceso de mejora que ayude a preservar y cuidar el planeta desde la enseñanza del docente hacia el estudiante, quien es un actor importante que podrá replicar lo aprendido desde las dimensiones ambientales en su hogar y con las demás personas con las que se relacione, presentando interés, compromiso y preocupación de lo que pueda suceder con el planeta y la biodiversidad que hace parte de él.

LaUNESCO(Organización de las naciones unidas para 
la educación la ciencia y la cultura) a finales de la década de los sesenta realizó un esfuerzo por estudiar las formas de incluir el tema ambiental como recurso educativo. Por lo cual solicitó a la Oficina Internacional de Educación (OIE) un estudio comparativo sobre la manera de abordar los temas del medio ambiente en la escuela, que pretendía detectar cuáles eran las actividades educativas que se realizaban en los países. Esta investigación mencionó en sus resultados la necesidad de abordar la temática ambiental desde una perspectiva transversal, criterio que luego sería uno de los principios de la EA (Educación ambiental). Sin embargo, y debido al contexto en el que se realiza el estudio, considera a la EA (Educación ambiental) como escolarizada, es decir, diseñada y ejecutada desde las instituciones educativas (Novo, 1998, p. 14).

El reto que tiene la Educación Ambiental es grande, ya que en el día a día surgen nuevas problemáticas $\mathrm{y}$ conflictos frente a la crisis ambiental por la que está pasando el planeta. El papel que tienen las instituciones educativas en sus procesos pedagógicos es el de resolver esas interrogantes que nacen frente a esas problemáticas, permitiéndole al estudiante aportar a la solución de estas, formándolos en competencias ciudadanas que les permitan contribuir y aportar su granito de arena en la preservación del ambiente, y siendo garantes del cuidado de este para el presente y las generaciones futuras. indican:

Velásquez y Flórez

El concepto y las prácticas en EA, por tanto, no se agotan en la siembra de árboles, el reciclaje, la recolección de basuras, las campañas educativas y la celebración de fechas ambientales, como suele suceder en muchos casos. Están relacionadas también con la interrelación humana, con las personas de la sociedad (familia, compañeros de estudio, de trabajo, amigos, entre otros) y con todos los demás seres vivos $\mathrm{y}$ elementos no vivos que conforman nuestro planeta. Se destaca que, dentro de estos procesos, la formación ambiental se preocupa no sólo por el cuidado de los ecosistemas, sino también por el autocuidado, del cuidado de nuestro hogar, de nuestro lugar de estudio, de trabajo y de los espacios sociales de interacción en general.

La EA es una respuesta a las problemáticas que se presentan en el ambiente, es una práctica pedagógica 
que da respuesta a las amenazas que se dan por el mal uso y aprovechamiento desmesurado de los bienes naturales $\mathrm{y}$ por los conflictos que afectan la vida de los seres vivos que habitan el planeta. Esta pedagogía se fundamenta en la educación escolar para que los estudiantes tengan una experiencia de compromiso y responsabilidad hacia el planeta.

\section{La Asignatura de Ciencias Naturales y el Proyecto Ambiental Escolar (PRAE)}

\section{El Proyecto Ambiental Escolar} (PRAE) permite el desarrollo de un proceso pedagógico y educativo que gire en torno a la investigación e intervención entre los actores que hacen parte de la comunidad educativa en procesos relacionados con temas ambientales, este proyecto debe estar incluido en el Proyecto Educativo Institucional (PEI) como eje transversal, para que en todas las asignaturas del plan de estudios se aborde la dimensión ambiental y no recaiga la responsabilidad solamente en el área de ciencias naturales. El PRAE es un proyecto que desde las aulas y en toda la institución educativa se vincula en la solución de problemáticas y conflictos ambientales que afectan el contexto de la institución y el territorio en donde esta se encuentra ubicada, generando espacios de reflexión, pensamiento crítico, solidaridad y tolerancia con fines a la mejora de la calidad de vida de las personas y de los demás seres vivos que habitan este entorno.
Según Fonseca y Ussa (2011):

"Toda la comunidad debe estar inmersa en la participación del desarrollo del PRAE, dando existencia al Comité Ambiental Escolar, que lidera la formulación, implementación seguimiento y evaluación del PRAE. Para esta directriz, un PRAE debe tener un propósito y unos objetivos, en su estructura un esquema conceptual que evidencie la relación de la sociedad con el ambiente, que dé soporte para tomar decisiones, que sea flexible, práctico y conste de una evaluación general e integrada".

Desde la fase diseño del PRAE las instituciones educativas deben realizar un diagnóstico de necesidades ambientales que se presenten, identificando las problemáticas ambientales relevantes para la comunidad educativa y de influencia, sean estas internas o externas al contexto institucional, para pensar en una propuesta pedagógica para incluirlas en las dimensiones que aborda el plan de estudios para trabajar una transversalidad basada en la resolución de problemas, abriendo un dialogo de saberes entre las distintas disciplinas o áreas para interpretar y generar procesos de investigación educativos de los cuales se puedan desglosar los otros proyectos transversales establecidos en el PEI.

Las estrategias, programas, acciones o alternativas que se propongan 
desde el PRAE para la intervención en estos aspectos ambientales identificados deben estar orientados a la búsqueda de una participación activa de toda la comunidad educativa, ya que esta se encuentra implícita en la generación de ciertos conflictos y problemáticas ambientales, así mismo se puede realizar una reflexión y lectura crítica del contexto para reconocer las dinámicas naturales, culturales y sociales que puedan minimizar, mitigar y dinamizar estas problemáticas y que den paso a la generación de escenarios para presentar alternativas de solución frente a la preservación, cuidado del ambiente y de los recursos naturales.

"La EA es una educación
que debe permitirnos
aprender a volver a habitar
nuestro planeta juntos,
nosotros los seres humanos
y con otras formas de vida
que comparten y conforman
nuestro entorno, rehabitar
colectivamente nuestros
espacios de vida" (Sauvé,
2010).

De esta manera, este proyecto transversal permite la articulación de diferentes saberes abordando la transversalidad con las demás áreas del plan de estudio, no debe recaer solo la responsabilidad en las ciencias naturales la cual aborda otros temas propios del desarrollo de su saber académico. Los estudiantes tienen un papel muy importante en dentro del PRAE y de la aplicación de la Educación Ambiental ya que son ellos los protagonistas del proceso educativo, el interés que presenten y la motivación que tengan frente al desarrollo de actividades ambientales permitirá llevar con éxito la ejecución de los planes trazados desde este y todos los demás proyectos transversales que se desarrollan en la institución educativa.

El PRAE abre espacios para el desarrollo de la investigación si se tiene en cuenta que su objeto es la formación para la comprensión de las problemáticas $\mathrm{y} / \mathrm{o}$ potencialidades ambientales, a través de la construcción de conocimientos significativos que redunden en beneficio de la cualificación de las actitudes y de los valores, en el marco de una formación ética y responsable frente al manejo adecuado del ambiente (competencias ciudadanas). (Torres, 1996)

Este dialogo de saberes favorece la articulación y la lectura de conceptos desde diferentes reflexiones y pensamiento crítico lo que enriquece el proceso de investigación que puede desarrollar el estudiante desde la Educación Ambiental, ya que se le brindan las herramientas para que pueda encontrar soluciones a problemáticas ambientales, a proponer alternativas de intervención sobre estas ya que habita el entorno y se desvuelve en este a través de sus actividades cotidianas de manera individual y colectiva, se realiza un reconocimiento del ser como individuo que hace parte del ambiente y que lo comparte con otros seres vivos entre fauna y flora, generando un aprendizaje significativo que le obliga al estudiante y a toda la comunidad 
educativa a preguntarse y a evaluar cómo es su forma de relacionarse con el ambiente y cuáles son los hábitos y comportamientos que está teniendo para preservar, cuidar y valorar el ambiente y los recursos naturales.

\section{Metodología}

El presente artículo de reflexión pretende serunaherramientaquepermita el analizar, comprender y entender la importancia de la Educación Ambiental en estudiantes de básica y media en este caso tomando tres instituciones educativas en El Espinal (Tolima) desde una mirada y un pensamiento crítico, reflexivo y académico, identificando las motivaciones, intereses, dificultades, falencias y actores que intervienen en el proceso educativo desde lo ambiental, en una conceptualización teórica y práctica en el desarrollo de la Educación Ambiental, utilizando una metodología que permita hallar respuestas estándares a través de un instrumento elaborado y aplicado a estos mismos, conociendo la opinión de los estudiantes frente al tema del cual nace esta investigación

Los autores Blasco y Pérez (2007:25), señalan que la investigación cualitativa estudia la realidad en su contexto natural y cómo sucede, sacando e interpretando fenómenos de acuerdo con las personas implicadas. Utiliza variedad de instrumentos para recoger información como las entrevistas, imágenes, observaciones, historias de vida, en los que se describen las rutinas y las situaciones problemáticas, así como los significados en la vida de los participantes.

La técnica de investigación que se utiliza es la encuesta y el instrumento el cuestionario de preguntas, el cual es aplicado a los estudiantes de los grados noveno, decimo y undécimo de las tres instituciones educativas. Se toma el $10 \%$ de la población estudiantil como muestra y los criterios para la selección de los estudiantes son la experiencia estudiantil que poseen algunos, al ser antiguos en la institución; además, están en una edad en la que se proyectan como próximos universitarios, son más centrados, maduros y participan en actividades institucionales, y se convierten en apoyo docente frente a grados inferiores.

\section{Discusión y Resultados}

Para el desarrollo del análisis de esta investigación se realizó un proceso de compresión y descripción en la importancia que tiene la Educación Ambiental en los estudiantes de las tres instituciones educativas abordadas, se encuestaron 30 estudiantes de los grados noveno, decimo y undécimo por institución educativa para la realización del respectivo cuestionario.

Entre las preguntas realizadas encontramos las siguientes:

1. ¿Considera que la Educación Ambiental es importante para su formación académica y personal?

2. ¿Ha tenido formación/ capacitación en temas ambientales?

3. ¿Considera usted que en todas las asignaturas (Matemáticas, Ciencias Naturales, Ciencias Sociales, Castellano, Inglés, Educación Religiosa, Educación Artística...) trabajan aspectos ambientales?

4. ¿Participa de manera permanente en actividades de Educación Ambiental ejecutadas desde 
el PRAE de la institución educativa?

Los estudiantes calificaban las anteriores preguntas bajo cada uno de los siguientes enunciados de acuerdo con su conocimiento o percepción, desde 1 (el peor nivel) hasta 4 (el mejor nivel).

\section{1: Nada 2: Muy poco 3: Poco 4: Mucho}

A continuación se realizara en análisis y la discusión frente a las respuestas que dieron los estudiantes de Institución Educativa Sector Urbano 1 (IESU1), Institución Educativa Sector Urbano 2 (IESU2) e Institución Educativa Sector Rural 3 (IESR3).

\section{Institución Educativa Sector Urbano 1 (IESU1)}

En esta institución educativa se presenta que los estudiantes de los diferentes grados han tenido una formación académica y capacitación en temas ambientales, desde el área de ciencias naturales principalmente, en la cual a través de la temáticas que se desarrollan durante el año académico se abren espacios en los que se celebran fechas ambientales pero de igual manera se realizan actividades teórico prácticas que permite comprender algunas situaciones ambientales que se presentan en la comunidad educativa $\mathrm{y}$ en la cual pueden intervenir desde su rol como estudiantes, así mimo ellos consideran que la Educación Ambiental es importante para su formación académica y personal ya que se encuentran preocupados por los daños que recibe en planeta por diferentes conflictos ambientales, así mismo comunican compromiso, interés y disposición para cuidar y proteger el ambiente y a la biodiversidad.

Por parte de los estudiantes haya un compromiso e interés frente a la Educación Ambiental ellos consideran que no se presenta una transversalidad ya que en las diferentes asignaturas que ven en su plan de estudios poco se trabajan o se abordan temas ambientales, cuando desde el currículo se puede trabajar las dimensiones ambientales de manera conjunta, estableciendo un aprendizaje basado en problemas, abordando la realidad del contexto del estudiante y la comunidad educativa frente al abordaje de necesidades y situaciones ambientales que se presenten.

Al momento de conocer la participación que tiene los estudiante frente a las actividades de Educación Ambiental la respuesta de la mayoría es que muy poco lo hace, pueda que participen en las actividades ambientales que se desarrollan en el calendario académico, pero no se sienten integrados y tenidos en cuenta frente al abordaje de diferentes temas ambientales que se podrían trabajar desde la institución, los estudiantes se interesan por un aprendizaje más practico desde la Educación Ambiental, aunque en ocasiones lo que falta también es un docente que genere espacios de aprendizaje significativo y que el apasione el trabajar por el ambiente inculcando a los estudiantes esto mismo, así se podrían mejorar los hábitos, comportamientos y actitudes de estos frente a la relación que tienen con el ambiente. 
Institución Educativa Sector Urbano 2 (IESU2)

En esta institución educativa los estudiantes consideran que tienen poca formación académica $\mathrm{y}$ capacitación en temas ambientales, reconociendo algunos vacíos en este aspecto pero considerando que para ellos la Educación Ambiental es muy importante en su formación académica y personal, presentando un interés y disposición frente actividades ambientales que se desarrollan durante el año académico en la institución y la ejecución de proyectos transversales que tengan como línea practica lo ambiental, el ambiente es un tema que les preocupa y están prestos a aportar en la vigilancia, control y protección de los recursos naturales, así mismo cuidando el ambiente en su comunidad educativa y en el contexto si se llegase a presentar situaciones donde se genere un conflicto ambiental.

Se presenta un vacío académico por parte de la institución educativa y en específico por la gestión docente ya que los estudiantes consideran que poco se trabajan temas ambientales en las asignaturas que ven en el plan de estudios, lo ambiental lo relacionan directamente a las ciencias naturales, cuando es indispensable romper ese tabú en que todo lo que tenga relación con el ambiente debe ser responsabilidad de esta asignatura o del Proyecto Ambiental (PRAE), cuando desde los lineamientos del Ministerio de Educación se espera trabajar la transversalidad y en este caso en específico la Educación Ambiental, la cual es un área del conocimiento que permite trabajar desde las diferentes asignaturas en la propuesta de situaciones reales y actuales en las que se vea la crisis ambiental y la afectación que está teniendo el ambiente y la naturaleza frente a las distintas actividades productivas que mueven la economía del país pero que consigo lleva el daño a los ecosistemas con fines de satisfacer las necesidades de la población, esto es lo que se puede presentar al estudiante desde el dialogo de saberes en las diferentes asignaturas para que el cuándo pueda y tenga la oportunidad de intervenir de manera positiva en la preservación del ambiente lo haga.

Los estudiantes consideran y responden que poco son tenidos en cuenta frente a la realización y participación que podrían tener frente a situaciones y actividades de Educación Ambiental para el abordaje de las diferentes necesidades ambientales que se puedan presentar en la institución educativa y en el contexto. Los docentes deben tener en cuenta y considerar importante al ambiente desde su labor académica, no cumplir simplemente con los que solicite la norma y la institución, deben preparar y enseñar a los estudiantes en buenos hábitos para relacionarse con el ambiente e instruirlos para que puedan intervenir y dar respuesta a las problemáticas y conflictos ambientales en los que se puedan ver inmersos, ya que ellos son las generaciones futuras, que tal vez puedan hacer un cambio frente a la crisis ambiental que se presenta en el planeta. 
Institución Educativa Sector Rural 3 (IESR3)

Esta institución educativa se encuentra ubicada en el sector rural de El Espinal (Tolima) los estudiantes presentan unas respuestas bastante amigables $y$ alentadoras frente a la encuesta realizada, ya que ellos y la institución se encuentra en contacto directo con la naturaleza y la biodiversidad, los estudiantes consideran que tienen formación académica y capacitación en temas ambientales, y de igual piensan $\mathrm{y}$ creen que la Educación Ambiental es importante para su formación académica y personal, estas respuestas dan a entender como el sector rural entiende $\mathrm{y}$ permanece en relación armoniosa para con el ambiente, en esta institución se realizan actividades académicas relacionadas con la agricultura, actividades que son desarrolladas por los estudiantes de una manera positiva y bien recibida, desde la experiencia que tienen ellos a nivel personal ya que las familias de donde provienen en la mayoría de los casos mueve la economía del hogar a través de los proceso agrícolas que se desarrollan en el sector en donde se encuentra ubicada la institución educativa.

Se encuentra un resultado interesante en esta institución educativa la cual estando ubicada en el sector rural los estudiantes consideran que poco se trabajan temas ambientales en la institución, desde una mirada practica se reconoce en el trabajo de campo realizado al momento de aplicar las encuestas que los estudiantes realizan actividades relacionadas con el sector agrícola, pero no se presenta una transversalidad de la Educación Ambiental, ya que desde las diferentes asignaturas no se aborda y se especifica la dimensión ambiental, contando y dejando pasar la oportunidad de desarrollar espacios de aprendizaje con los estudiantes en los cuales se aborden necesidades, problemáticas $y$ conflictos que se presenten en el ambiente y en contexto real en el que se encuentran ellos, ya que la zona en donde se está ubicada la institución al ser agrícola presenta varias situaciones desde la generación de varios factores contaminantes al no realizar buenas prácticas de agricultura frente al uso de químicos para los cultivos y la contaminación de las fuentes hídricas y demás, igualmente el daño al suelo por el uso de maquinaria y alteración físico química para la siembra, solo por mencionar dos aspectos que se podrían abordar con los estudiantes y cuales son visibles a la realidad de ellos. Permitiendo generar espacios de reflexión y debate para cuando ellos tengan bajo su rol y desempeñen tal vez su actividad laboral y económica en el sector agrícola puedan aportar al cuidado y preservación del ambiente con unas buenas prácticas de agricultura amigables con la naturaleza.

Se presenta que los estudiantes de esta institución consideran que poco son tenidos en cuenta frente a la realización de actividades de Educación Ambiental, reconociendo el sector en el que se encuentra la institución educativa y la riqueza ambiental con la que cuenta, es interesante toda actividad académica que realizan con un énfasis agrícola que es hacia donde se proyecta la formación que reciben, pero hace falta que los docentes y 
la comunidad educativa en general redireccione los procesos académicos hacia un dialogo de saberes en donde el estudiante pueda participar, muchos tienen buenas ideas que merecen ser escuchadas y tal vez desarrolladas frente situaciones ambientales que observen y donde les interese intervenir como agentes ambientales preocupados por la preservación de la naturaleza y del entorno en el que nacieron y desde el cual se han formado como personas.

\section{Conclusiones}

Se observó la importancia que tiene la Educación Ambiental en los estudiantes de las tres instituciones educativas para la vida académica y personal de ellos, presentando vacíos en algunos momentos en temas ambientales, ya que se necesita una formación académica constante que involucre e informe al estudiante de la realidad de los conflictos y problemas ambientales por los que pasa el planeta, el país y su región en específico, brindando la oportunidad de reflexionar y pensar cual podría su rol y aporte para cuidar, proteger y preservar el ambiente.

Se presenta un interés, compromiso y preocupación por parte de los estudiantes sobre el cuidado del ambiente y como la Educación Ambiental les puede permitir conocer y aprender a través de diferentes herramientas prácticas y teóricas para el desarrollo y ejecución de programas, proyectos y generación de alternativas o soluciones frente al aprendizaje de hábitos, comportamientos, valores $\mathrm{y}$ una cultura ambiental para relacionarse de manera correcta con la naturaleza.
Se reconoce el rol que tiene el docente en el compromiso que debe adquirir para capacitarse en temas ambientales y poderles ofrecer a los estudiantes espacios de aprendizaje en los que se pueda generar la reflexión y el pensamiento crítico frente a la crisis ambientales por la que pasa el planeta y enseñando como se puede aportar a la mitigación y control de problemáticas ambientales que se puedan analizar desde la realidad del contexto de los estudiantes y en la que ellos puedan intervenir y dar solución, comprendiendo que ellos también son agentes replicadores de información y lo que aprendan desde la Educación Ambiental en la institución lo podrán aplicar en el hogar son sus familias y con las personas con las que se relacione externamente a la institución educativa.

Las instituciones educativas tiene dos ejes importantes para enseñar la Educación Ambiental está el Proyecto Ambiental Escolar (PRAE) y la asignatura de Ciencias Naturales, ambos espacios de aprendizaje involucran al estudiante frente a las dinámicas naturales y sociales que se desarrollan frente a diferentes situaciones que se presentan y que afectan al ambiente, pero no se debe dejar la responsabilidad solamente a estos dos procesos académicos, la Educación Ambiental debe ser transversal y permitir un diálogo de saberes entre las diferentes asignaturas del plan de estudios, ya que desde varias dimensiones y puntos de vida se pueden fortalecer las habilidades y competencias de los estudiantes para hacerlos agentes de cambio ambiental fortaleciendo en ellos una conciencia y cultura ambiental para que puedan aportar a la 
mejora del ambiente, la preservación de la naturaleza y la biodiversidad a través de buenas prácticas ambientales en sus actividades cotidianas.

\section{Referencia Bibliográfica}

Alea, A. (2008). Breve historia de la educación ambiental: del conservacionismo hacia el desarrollo sostenible. Recuperado de: $\quad$ http://ftp.murciaeduca. es/programas educativos/ $\mathrm{Nuevo1/RECesenred/}$ historiaeducacionambiental.pdf

Blasco, J. Pérez, J. (2007). Metodologías de investigación en las ciencias de la actividad física y del deporte: ampliando horizontes. Recuperado de: https://rua.ua.es/dspace/ bitstream/10045/12270/1/blasco. pdf

Carrasco, M. (1994). Dimensión ambiental y proceso educativo: unas propuestas y propósitos ambientales. Serie de documentos especiales. MEN. Seminario internacional. $\mathrm{La}$ dimensión ambiental y la escuela, 97-119.

Flórez Espinosa, G.M, Pino Perdono, F.M, Velásquez Sarria, J.A., \& Gálvez Cubides D.J. (2019). Caracterización de concepciones de educación ambiental en estudiantes de últimos semestres de Licenciatura en Educación Básica con Énfasis en Ciencias Naturales y Educación Ambiental de tres universidades de Colombia. Ibagué: Universidad del Tolima.

Hernández, R., Fernández, C. y Baptista, P. (2014). Metodología de la investigación ( $6^{\mathrm{a}}$ ed.). México: McGrwall Hill Education.
Hernández, R., Méndez, S. y Mendoza, C. (2014). Capítulo 1. En Metodología de la investigación, página web de Online Learning Center. Consultado en la red mundial el 29 de abril de 2015

Ministerio de Ambiente y Desarrollo Sostenible. (2014). Código Nacional de Recursos Naturales Renovables y de Protección al Medio Ambiente. Decreto ley 2811 de 1974. Bogotá D.C.

Ministerio de Educación Nacional. (1994).

Decreto 1743. Bogotá D.C.

Ministerio de Educación Nacional. (1994). Decreto 1860 de 1994. Bogotá D.C.

Novo M. (1998).Educación ambiental. Base ética, conceptual y metodológica. Artículo citado en http://www.ehu.es/cdsea/web/ revista/numero $1 / 0103$ macedo. pdf

Sauvé, L. (2010). Educación cientifica y educación ambiental. Un cruce fecundo. Universidad Du Quepbec \& Montreal

Sepúlveda Gallego, L.h (2007) Proyectos Ambientales Escolares de Manizales. Revista Luna Azul, 24, $15-22$

Vargas, C. \& Estupiñán, M. (2012). Estrategias para la Educación Ambiental con escolares pobladores del páramo Rabana (Boyaca). Luna Azul. 